\title{
Surface-gel-conversion synthesis of submicron-thick MFI zeolite membranes to expedite shape-selective separation of hexane isomers
}

\author{
Huazhen Rong ${ }^{1}$, Gaimei Wang ${ }^{1}$, Jiaxu Yan ${ }^{2}$, Xiaoqin Zou ${ }^{1 *}$ and Guangshan Zhu
}

\begin{abstract}
Ultrathin zeolite membranes are of paramount importance in accelerating gas transport during membrane separation, and lowering down their membrane thicknesses to submicron scale is deemed to be very challenging. Herein, we develop an advanced approach of surface gel conversion for synthesis of submicron-thick pure silica MFI (silicalite-1) zeolite membranes. Viscous gel is prepared by finely adjusting the precursor composition, enabling its reduced wettability. The unfavorable wetting of the support surface can effectively prevent gel penetration into alumina support voids. Aided by the seeds, the surface gel is directly and fully crystallized into an MFI zeolite membrane with minimal water steam. A membrane with a thickness of $500 \mathbf{n m}$ is successfully acquired and it is free of visible cracks. Additionally, the as-synthesized membranes exhibit rapid and selective separation of hexane isomers by virtue of unprecedentedly high $n$-hexane permeance of $24.5 \times 10^{-7} \mathrm{~mol} \mathrm{~m}^{-2} \mathrm{~s}^{-1} \mathrm{~Pa}^{-1}$ and impressive separation factors of 13.3-22.6 for $n$-hexane over its isomers. This developed approach is of practical interest for sustainable synthesis of high-quality zeolite membranes.
\end{abstract}

Keywords: zeolite synthesis, silica gel chemistry, thin membrane, hexane separation, isomer selectivity

\section{INTRODUCTION}

Zeolite membranes are of immense potential in separation technology owing to continuous working mode and energy efficiency. They are made of crystalline zeolites with uniform and molecule-level pores which are interconnected for mass transport [1]. Among different zeolites, MFI-type (ZSM-5 and silicalite-1) zeolite is a representative in membrane application because it has $5.5 \AA$ multidimensional pores for sieving molecules of industrial interest (i.e., $\mathrm{H}_{2} / \mathrm{N}_{2}$ ) and tunable adsorption properties favorable in separating important petrochemical compounds (i.e., alkanes) [2,3]. Although some progress has been made to enhance membrane performances by chemical synthesis (i.e., microwave irradiation) [4] and membrane engineering (i.e., crystal orientation) [5], MFI zeolite membranes still can hardly substitute existing technologies (i.e., distillation) due to their high fabrication cost [6-9]. One feasible way to compensate the high capital investment is to increase the flux in the separation process while maintaining the selectivity [10]. This means that the membrane thickness should be substantially reduced because the flux is inversely proportional to the thickness.

To achieve ultrathin (i.e., submicron in thickness) zeolite membrane is very challenging, evidenced by longtime endeavors from numerous scientists and engineers [11-17]. Currently developed synthesis methods for MFI thin films are categorized in the following: (1) controlled crystallization by additives such as fluoride ion and organic modifier. These additives have to be elaborately selected to slow down crystallization or to direct in-plane crystal growth $[11,12]$. (2) Support modifications by void plugging. Support pretreatments are sophisticated, requiring careful impregnation of extra substances into support voids $[13,14]$. (3) Seeding with zeolite nanosheets. Secondary growth of nanosheets-based seed layer can yield zeolite thin films [15-17]. In this method, generally speaking, very special and complex organic templates are needed for synthesis of MFI nanosheets.

The above syntheses are usually carried out in hydrothermal systems which require a lot of synthesis solution and produce a large amount of wastes. Alternative to hydrothermal synthesis, dry-gel conversion exhibits several advantages; for example, a small quantity of gel is

\footnotetext{
${ }^{1}$ Faculty of Chemistry, Northeast Normal University, Changchun 130024, China

${ }^{2}$ Key Laboratory of UV-Emitting Materials and Technology of Ministry of Education, Northeast Normal University, Changchun 130024, China

* Corresponding author (email: zouxq100@nenu.edu.cn)
} 
needed for the membrane synthesis, zeolites are crystallized in high yields, and no liquid/solid wastes are disposed [18-22]. However, defects or cracks are prone to form in the membrane due to volume shrinkage in the gel-to-zeolite crystallization [18-23]. To acquire acceptable selectivity, the membranes have to be grown thick, which inevitably decreases the flux [21,24]. Thus, advanced methods are desirable for the green synthesis of highly permeable and selective MFI membranes. Herein, we develop a surface-gel-conversion approach, which can ideally meet the criteria of high permeability, good selectivity and sustainability for modern membrane technology. This approach involves first deposition of a thin layer of amorphous silica gel on the seeded support and its subsequent transformation into a membrane (Scheme 1). Hence, the top gel layer is determinative to zeolite membrane because the membrane thickness is a function of the gel thickness. In this regard, the microstructure of gel precursor is tuned to regulate its wettability and accordingly to precisely control the gel thickness. Assisted with minimal steam, the whole gel is fully crystallized to silicalite-1 zeolites (pure-silica MFI zeolite) on an alumina support, leading to a commensurately gel-thick membrane. The membrane thickness can be facilely reduced to sub- $1 \mu \mathrm{m}$ by decreasing the gel layer. The membranes after additional growth are free of cracks and capable of separating hexane isomers with unprecedented permeance and impressive separation factor.

\section{EXPERIMENTAL SECTION}

\section{Chemicals}

Tetrapropylammonium hydroxide (TPAOH, 25\% in wa-

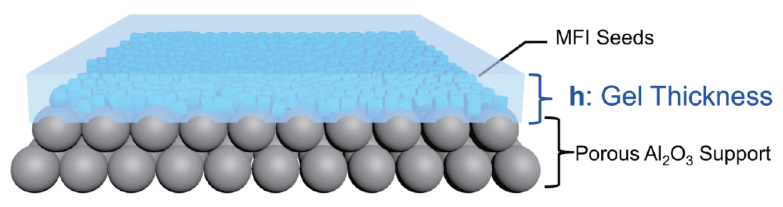

$$
\begin{aligned}
& \text { Surface Gel Conversion } \\
& \text { Temperature, Time }
\end{aligned} \quad h^{\prime}=f(h)
$$

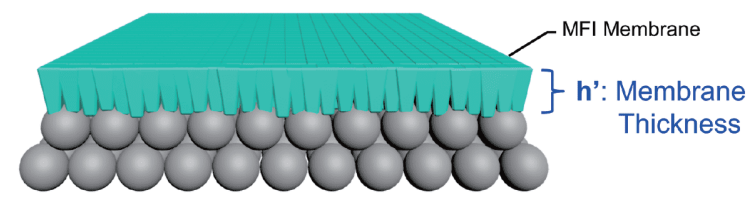

Scheme 1 Schematic illustration of the surface-gel-conversion method for synthesis of the MFI zeolite membrane. ter, Tianjin GuangFu Fine Chemical, China), tetraethyl orthosilicate (TEOS, 98\%, Sinopharm Chemical Reagent Co., Ltd, China), and hexane isomers ( $n$-hexane (nHEX), 2-methylpentane (2MP), 3-methylpentane (3MP), 2,3dimethylbutane (23DMB), 2,2-dimethylbutane (22DMB), $98.0 \%$, TCI) were purchased from the corresponding companies. Deionized water was used as the solvent. Porous $\mathrm{Al}_{2} \mathrm{O}_{3}$ discs $(28 \mathrm{~mm}$ in diameter, $3 \mathrm{~mm}$ in thickness, $40 \%$ in porosity, $1-3 \mu \mathrm{m}$ in pore size) were purchased from Guangdong Yuli Electronic Co., Ltd, China. These discs were polished with sand papers of 500, 1000 and 1500 mesh, washed with deionized water and then dried at $85^{\circ} \mathrm{C}$ for $5 \mathrm{~h}$.

\section{Syntheses of MFI seeds and membranes}

MFI seeds in nanoscale were prepared with a synthesis solution in mole ratio of 1.0TPAOH:3.64TEOS: $33.8 \mathrm{H}_{2} \mathrm{O}$ according to our published recipe [25]. Briefly, $8.0 \mathrm{~g}$ TPAOH solution (25\%) was mixed with $7.46 \mathrm{~g}$ TEOS in a 25-mL polypropylene (PP) bottle to form a clear solution under stirring for $12 \mathrm{~h}$ at room temperature. This solution was transferred into a $25-\mathrm{mL}$ Teflon-lined autoclave and heated at $120^{\circ} \mathrm{C}$ for $12 \mathrm{~h}$. After cooling, the suspension was washed by repetitive centrifugations $\left(18,000 \mathrm{r} \mathrm{min}^{-1}\right.$ and $20 \mathrm{~min}$ for each run) until the supernatant was almost neutral. The seed solution of $1.0 \mathrm{wt} \%$ was prepared by dispersing seeds in deionized water. Seeding $\mathrm{Al}_{2} \mathrm{O}_{3}$ disc was carried out by immersing half height of the disc into $1.0 \mathrm{wt} \%$ seed solution with the polished surface downwards for $30 \mathrm{~s}$. The wet disc was dried at $85^{\circ} \mathrm{C}$ for $30 \mathrm{~min}$ and the seeding procedure was repeated twice. The seeded surface was smoothed by finger with glove. The seeded $\mathrm{Al}_{2} \mathrm{O}_{3}$ disc was calcined at $550^{\circ} \mathrm{C}$ for $6 \mathrm{~h}$ (heating ramp of $1.0^{\circ} \mathrm{C} \mathrm{min}^{-1}$ ). MFI membranes were synthesized by the surface-gel-conversion method. The gel was prepared with a chemical composition of 1.0TPAOH:16.1TEOS: $580 \mathrm{H}_{2} \mathrm{O}$. Typically, $1.225 \mathrm{~g}$ TPAOH solution (25\%) was mixed with $14.85 \mathrm{~g}$ water in a $25-\mathrm{mL}$ PP bottle, and then $5.05 \mathrm{~g}$ TEOS was added in the bottle, with additional stirring for $48 \mathrm{~h}$ at room temperature. For comparison, reference solutions were prepared with compositions of 1.0TPAOH:16.1TEOS: $2765 \mathrm{H}_{2} \mathrm{O}$ (labeled as Ref.1) and 1.0TPAOH:3.2TEOS: $117 \mathrm{H}_{2} \mathrm{O}$ (labeled as Ref.2). The gel was deposited on the seeded support by the spin-coating technique. About $2 \mathrm{~mL}$ of the gel was added on the seeded $\mathrm{Al}_{2} \mathrm{O}_{3}$ disc and this disc was spun at $1500 \mathrm{rmin}^{-1}(<1 \mathrm{~s}$ to reach this speed) for $10 \mathrm{~s}$ (temperature of $25 \pm 3^{\circ} \mathrm{C}$, relative humidity of $40 \% \pm 5 \%)$. The gel-coated disc was fixed horizontally on a sample holder and then placed in a $50-\mathrm{mL}$ autoclave 
with $0.2 \mathrm{~mL}$ water in the bottom. The autoclave was heated at $150^{\circ} \mathrm{C}$ in a static oven for $3 \mathrm{~d}$ for zeolite crystallization. The templates in the prepared membrane were removed by calcination at $550^{\circ} \mathrm{C}$ for $6 \mathrm{~h}$ with a heating rate of $1.0^{\circ} \mathrm{C} \mathrm{m^{-1 }}$ in air.

\section{Characterizations}

The sizes and zeta potentials of silica colloids were measured on a Nano ZS90 laser particle analyzer (Malvern Instruments, UK) at room temperature $\left(\sim 25^{\circ} \mathrm{C}\right)$. The silica concentration was adjusted around $100 \mu \mathrm{g} \mathrm{mL} \mathrm{L}^{-1}$ for the measurements. Fourier transform infrared (FT-IR) spectrum of freeze-dried gel was recorded on a Nicolet iS50 Fourier transform infrared spectrometer (Thermo Fisher Scientific, USA). Raman spectrum was recorded on the same sample using ultraviolet (UV) Raman spectroscopy (HORIBA Scientific, Japan), excited by $325 \mathrm{~nm}$ laser. X-ray diffraction (XRD) was performed on a Rigaku SmartLab X-ray diffractometer (Japan) with $\mathrm{Cu}-\mathrm{Ka}$ radiation of $\lambda=1.5418 \AA(40 \mathrm{kV}$ and $30 \mathrm{~mA})$. The membrane morphology was inspected with field-emission scanning electron microscopy (FE-SEM, SU8010, Hitachi, Japan), which was equipped with energy-dispersive X-ray spectroscopy (EDX). The contact angle between gel and support was measured after gel stabilization for $10 \mathrm{~s}$ on the instrument of CA-100 (Shanghai Innuo Precision Instruments Co., Ltd, China). The viscosity values were collected on a rotational viscometer of DV-1 (Shanghai Yueping Scientific Instrument Co., Ltd, China). The depth of gel penetration $\left(l_{\mathrm{g}}\right)$ was estimated according to the following equation:

$l_{\mathrm{g}}=\frac{M_{\mathrm{g}}}{\rho_{\mathrm{g}} \times A \times \varepsilon}$,

where $M_{\mathrm{g}}$ is the weight of the penetrated gel (penetration time is about $10 \mathrm{~s}), \rho_{\mathrm{g}}$ is the gel density $\left(\sim 1.0 \mathrm{~g} \mathrm{~cm}^{-3}\right), A$ is the top surface area of the support $\left(6.15 \mathrm{~cm}^{2}\right)$, and $\varepsilon$ is the support porosity (40\%).

\section{Membrane separation}

The separation property was examined by the permeation of hexane isomer vapor (see Fig. S1). Hexane (nHEX) and its isomers (2MP, 3MP, 23DMB, and 22DMB) were stored in a saturator which was controlled from -20 to $0^{\circ} \mathrm{C}$ by a cooling apparatus. The zeolite membrane was fixed in a membrane module and sealed with xyleneresistant O-rings. This module was connected to a gas flow system. The carrier gas of argon passed through the saturator and supplied to the feed side of the membrane module with a flow rate of $50 \mathrm{~mL} \mathrm{~min}{ }^{-1}$. The sweep gas of argon passed through the permeate side with a flow rate of $25 \mathrm{~mL} \mathrm{~min}^{-1}$. The feed-to-permeate pressure drop was kept at 1.2 bar by a pressure gauge and the module temperature was maintained at $100^{\circ} \mathrm{C}$ in a tubular furnace. The concentration of hexane or its isomer in feed or permeate was monitored by online gas chromatography (Techcomp GC7900) equipped with TM-PLOT $\mathrm{Al}_{2} \mathrm{O}_{3} / \mathrm{S}$ column $(30 \mathrm{~m} \times 0.53 \mathrm{~mm} \times 25 \mu \mathrm{m})$ and a flame ionization detector. $\mathrm{H}_{2}$ or $\mathrm{N}_{2}$ gas was detected on a thermal conductivity detector.

\section{RESULTS AND DISCUSSION}

A precursor solution with an initial mole composition of 1.0TPAOH:16.1TEOS: $580 \mathrm{H}_{2} \mathrm{O}$ (labeled as Gel) was optimized for the membrane synthesis. The silica chemical structure was studied by IR spectroscopy (Fig. 1a). IR spectrum contains strong bands at 1100, 800 and $460 \mathrm{~cm}^{-1}$, corresponding to asymmetric, symmetric and bending vibrations of siloxane bonds [26,27]. The presence of siloxane rings is confirmed by the Raman shift at $490 \mathrm{~cm}^{-1}$ (Fig. S2) [28]. Weak bands at 3630 and $965 \mathrm{~cm}^{-1}$ (Fig. 1a) can be assigned to stretching and bending vibrations of silanol groups. Broadening of IR band at $3430 \mathrm{~cm}^{-1}$ originates from hydrogen-bonded $\mathrm{Si}-\mathrm{OH}$ with $\mathrm{H}_{2} \mathrm{O}$. XRD pattern (Fig. S3) reveals the amorphous nature of the silica, consistent with superimposed Raman shifts of $450-490 \mathrm{~cm}^{-1}$ (Fig. S2). Reference precursors show the same band positions (Fig. 1a), indicating that they have the same chemical silica species, regardless of the addition of more water or concentrated TPAOH in solutions. Fig. $1 \mathrm{~b}$ presents particle-size distributions of silica in the three precursors measured by dynamic light scattering (DLS). It can be seen that all precursors contain dispersed and stable colloids and the majority of particles are around $1.5 \mathrm{~nm}$, showing that hydrolyzed silica after $48 \mathrm{~h}$ undergo similar sequential reactions of polymerization and particle growth. IR, Raman and DLS results reveal that silica particles in all precursors possess identical chemical structures and colloidal nature. Fig. 1c shows that the gel has the highest relative viscosity among the three precursors, indicating that the strongest interaction occurs between colloids in the gel. To unveil the underlying reason, the relative viscosities were investigated for different solutions by varying silica volume fraction $\left(V_{\mathrm{f}, \mathrm{SiO}_{2}}\right)$ and changing $\mathrm{pH}$. The viscosity (Fig. S4a) increases rapidly as more silica is added in the solution, in particular for the high volume-fraction precursors (e.g., $\left.V_{\mathrm{f}, \mathrm{SiO}_{2}}=0.03\right)$. High volume fraction means short particleparticle distance, resulting in strong inter-particle at- 

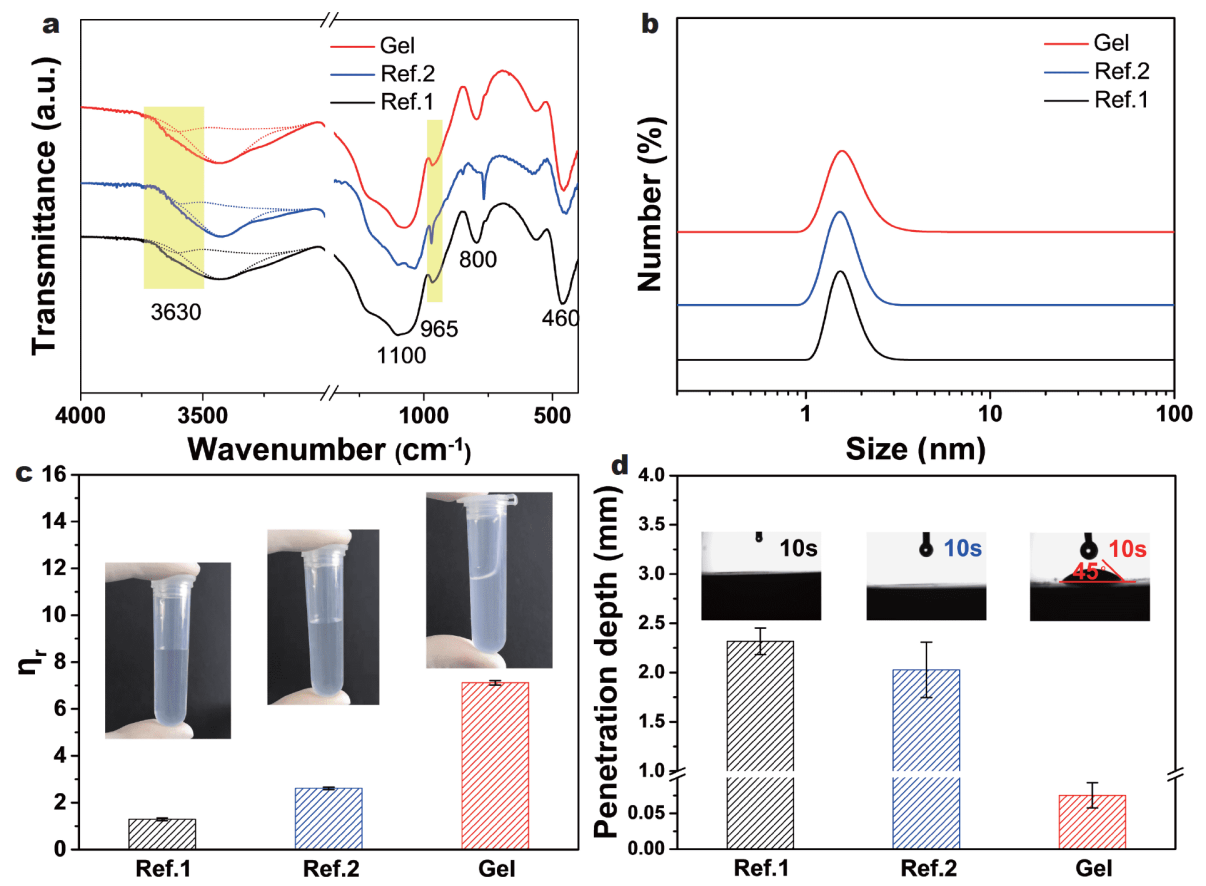

Figure 1 (a) IR spectra of freeze-dried gel and reference precursors (1.0TPAOH:16.1TEOS:2765 $\mathrm{H}_{2} \mathrm{O}$ and 1.0TPAOH:3.2TEOS:117 $\mathrm{H}_{2} \mathrm{O}$ for Ref.1 and Ref.2, respectively). (b) Particle sizes of silica colloids measured by DLS. (c) Relative viscosities of the gel and reference precursors (viscosity relative to water). The insets are optical pictures of the precursors. (d) Penetration depths of the gel and reference precursors on the seeded $\mathrm{Al}_{2} \mathrm{O}_{3}$ support (penetration time is $10 \mathrm{~s}$ ). The insets are contact angles of the precursors on the support.

traction via van der Waals force or hydrogen bonding [29]. The viscosity in Fig. S4b decreases when the solution has a high $\mathrm{pH}$ value. This can be rationalized by an increased repulsion between colloidal silica particles with high surface charges (zeta potentials of $-25,-26.5$ and $-38.6 \mathrm{mV}$ for Gel, Ref.1 and Ref.2 solutions) [29]. The high content and low $\mathrm{pH}$ result in extensive and strong interactions between small silica colloids in the gel. As a consequence, the wettability of the gel precursor could be reduced. To prove this hypothesis, contact angles of silica-containing water drops on the seeded supports were measured in Fig. 1d. A contact angle of $45^{\circ}$ is measured for the gel precursor. As comparison, contact angles of the other two precursors are approximately $0^{\circ}$. Higher contact angle describes less tendency of wetting the support [30], translating that the gel is difficult to penetrate into the support voids. A penetration depth of $75 \mu \mathrm{m}$ is mathematically determined for the gel (in $10 \mathrm{~s}$, suitable for spin coating), which is significantly lower than those for reference precursors $(2300$ and $2030 \mu \mathrm{m}$ for Ref.1 and Ref.2).

In this study, MFI zeolite seeds were used to assist the conversion of the surface gel for the membrane synthesis. Without seeds, isolated crystals are formed in the gel and loosely deposited on the $\mathrm{Al}_{2} \mathrm{O}_{3}$ support (Fig. S5). In contrast, by seeding the support with MFI nanocrystals, these crystals are inter-grown together after the secondary crystallization process and a compact membrane is yielded on the support (Figs 2 and 3). This promotion effect of seeds in zeolite growths of membranes is also observed in other studies $[3,21,23]$. Ref.1 and Ref.2 were also employed for the seeded syntheses of MFI membranes. It can be seen that the membrane prepared from Ref.1 is not continuous (Fig. S6) due to the very slow crystallization in dilute solution. In spite of membrane continuity achieved in Ref.2, the support pores are fully blocked by zeolite crystals, inevitably leading to an increase in membrane thickness (Fig. S6), consistent with the observation of precursor penetration depth (Fig. 1d). These findings show that the gel property and zeolite seeding are indispensable for obtaining high-quality membranes by surface gel conversion. In addition, the method of seedassisted surface gel conversion allows the feasibility to accurately control the membrane thickness. Fig. 2 displays the relationship between the gel weight and membrane thickness. It can be seen that the membrane thickness is proportionally dependent on the gel weight. This result demonstrates that the MFI zeolite membrane 


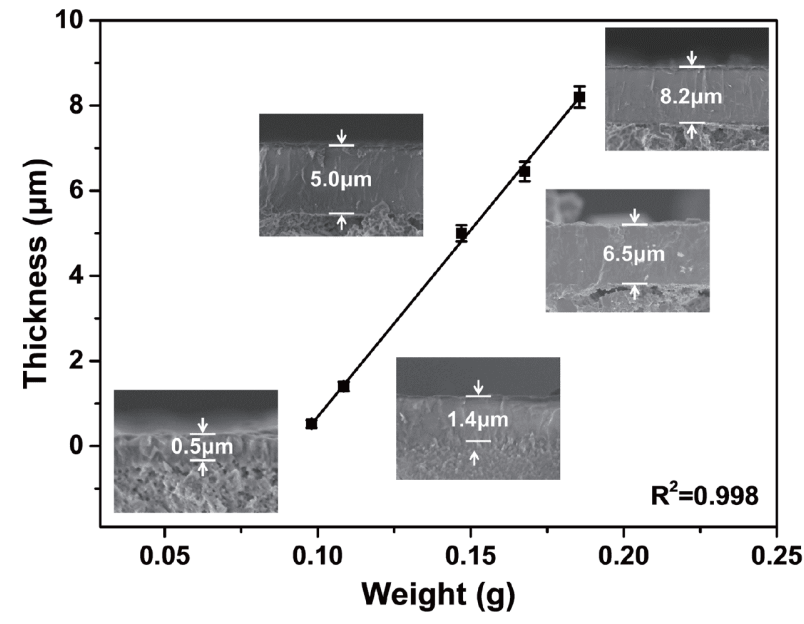

Figure 2 The thicknesses of MFI zeolite membranes as a function of the coated gel weights. The insets are SEM images of the prepared MFI membranes on porous $\mathrm{Al}_{2} \mathrm{O}_{3}$ supports.
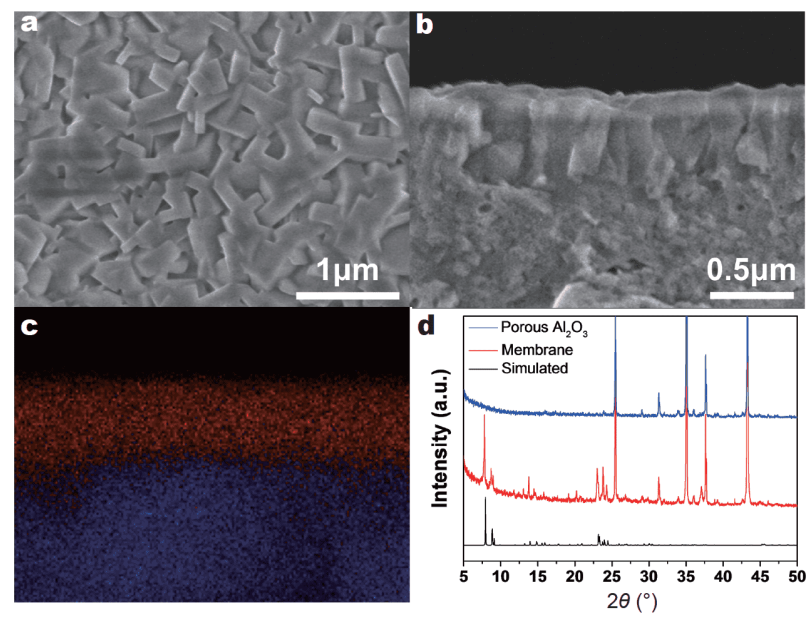

Figure 3 (a, b) Top and side views of SEM images of the synthesized MFI zeolite membrane, and (c) the corresponding EDX mapping (Si and $\mathrm{Al}$ elements are presented by red and blue colors, respectively). (d) XRD patterns of the simulated MFI zeolite, porous $\mathrm{Al}_{2} \mathrm{O}_{3}$ support and synthesized membrane.

can be facilely thinned down to $500 \mathrm{~nm}$ through conversion of comparably-thick surface gel. This approach can be extended to other supports, exemplified by transferring porous alumina to silicon substrate. Linear correlation is observed for membrane thickness in function of gel thickness which can be easily varied by the weight of surface gel deposited on the substrate (Fig. S7).

Thin MFI zeolite membranes were synthesized after optimization of the synthesis conditions. Fig. 3a, b show top and side views of SEM images for the MFI membranes. The crystals are well inter-grown to form a con- tinuous membrane and no cracks are inspected (Fig. 3a), showing that the obtained membrane is free of macroscopic defects. The membrane thickness is around $500 \mathrm{~nm}$, determined from the cross-sectional SEM image (Fig. 3b). The membrane-support interface can be clearly distinguished from the EDX mapping (Fig. 3c), resulting from negligible gel penetration into the alumina support. As seen from the XRD pattern in Fig. 3d, the prepared membrane exhibits characteristic MFI zeolite diffractions and is free of other zeolite impurities. The XRD pattern also reveals that the membrane has a random orientation of crystals, in agreement with the SEM inspections (Fig. 3a, b).

The crystallization habit of the MFI zeolite in the membrane was investigated by SEM. Nano-zeolites were applied for seeding a representative flat substrate of silicon (Fig. 4a), similarly to $\mathrm{Al}_{2} \mathrm{O}_{3}$ support. MFI nanocrystals were first deposited on silicon and then a thick gel layer $(\sim 3.5 \mu \mathrm{m})$ was intentionally coated on the seeds for a clear investigation. After $1 \mathrm{~h}$ crystallization, the individual seeds $(100 \mathrm{~nm})$ in spherical shape grow into larger crystals of $130 \mathrm{~nm} \times 240 \mathrm{~nm}$ by adsorbing silica nutrients from the top gel (Fig. 4b). In addition, the crystal growth proceeds faster horizontally than vertically,

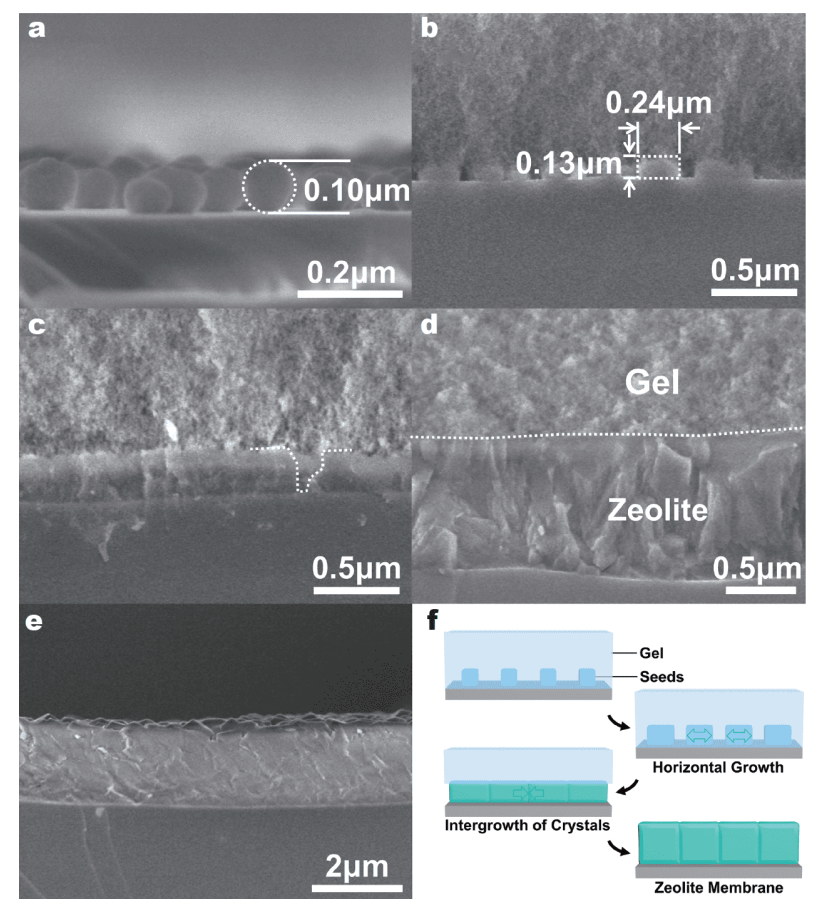

Figure 4 Side views of SEM images of the silicon-supported seed layer (a) and zeolite membranes after crystallization of $1 \mathrm{~h}$ (b), $2 \mathrm{~h} \mathrm{(c),} 4 \mathrm{~h}$ (d), $5 \mathrm{~h}$ (e) at $150^{\circ} \mathrm{C}$. (f) The possible growth process of the MFI zeolite membrane. The dash lines present a defect in zeolite film in (c) and the gel-zeolite interface in (d). 
evidenced by a larger crystal aspect ratio of $\sim 2.0$ (Fig. $4 \mathrm{~b}$ ) than that of $\sim 1.0$ for the seed (Fig. $4 \mathrm{a}$ ).

This is beneficial for healing crystal boundaries. As the crystallization progresses for $2 \mathrm{~h}$, zeolite crystals experience further growth and consequently merge into an inter-grown layer (Fig. 4c). At this stage, most crystals undergo preferentially vertical growth due to space limitation in the horizontal direction [25]. The crystallization process is briefly illustrated in Fig. 4f. As suggested in Fig. $4 \mathrm{f}$ and shown in Fig. $4 \mathrm{~d}$, some remaining defects (dash-lined area in Fig. 4c) can be eliminated by prolonging the crystallization time to $4 \mathrm{~h}$. The nutrients in the upper gel (indicated by dash line in Fig. 4d) were completely consumed after $5 \mathrm{~h}$ and an entirely continuous zeolite membrane with well inter-grown crystals was yielded on the substrate (Fig. 4e).

The above results encourage us to evaluate the separation performance of this thin silicalite-1 zeolite membrane. Prior to separation, the quality of the silicalite-1 membrane was examined by standard hydrogen and nitrogen gas permeation tests. The selectivity for hydrogen over nitrogen measured at $25^{\circ} \mathrm{C}$ and $1.2 \mathrm{bar}$ is 4.47 , larger than the Knudsen constant of 3.73 , supporting that the membrane is devoid of microscopic defects. Subsequently, the separation of hexane isomers was conducted under a standard Wicke-Kallenbach configuration using single or mixture vapor as the gas feed [31]. The gas permeation data at $100^{\circ} \mathrm{C}$ is compiled in Table 1 . The permeance of $24.5 \times 10^{-7} \mathrm{~mol} \mathrm{~m}^{-2} \mathrm{~s}^{-1} \mathrm{~Pa}^{-1}$ is measured for nHEX single gas. This is significantly larger than those for branched hexanes, translating to separation factors of 13.3-22.6. The separation factors follow the sequence of $13.7 \approx 13.3<21.5<22.6$ for $\mathrm{nHEX} / 2 \mathrm{MP}, \mathrm{nHEX} / 3 \mathrm{MP}$, $\mathrm{nHEX} / 23 \mathrm{DMB}$ and $\mathrm{nHEX} / 22 \mathrm{DMB}$, respectively, in coincidence with the trend of their molecular sizes (Table 1), indicative of size-selective separation of hexane in the MFI membrane. For comparison, the performances of all relevant MFI zeolite membranes are summarized in Fig. 5 [32-43]. As shown, this submicron membrane gives the highest permeance among all pertinent membranes. If the permeance is plotted versus the thickness (inset in Fig. 5), we have found that the nHEX permeance in general follows the decreasing trend with thickening the membrane. This finding soundly proves that reducing the membrane thickness is very effective in enhancing gas permeability. The separation factor exemplified by nHEX over 2,2-dimethylbutane is also quite good according to reported membranes, and it can be further improved by our coming effort in the future research.

When a binary gas is fed into the membrane, the gas permeance decreases and concurrently the separation factor increases in comparison with single gas, which can be interpreted by the competitive permeation mechanism of hexane isomers (nHEX, 2MP, 3MP, 23DMB, and 22DMB) [33]. The improved separation factor validates that the membrane is in good quality such as high continuity. To assess the practical application of this membrane, the separation test was carried out on a multicomponent mixture in volume composition of 13:20:18:19:30 for nHEX:2MP:3MP:23DMB:22DMB [44]. The permeance and separation factor were recorded as a function of time (Fig. 6). The permeance is very high initially at $6.0 \times 10^{-7} \mathrm{~mol} \mathrm{~m}^{-2} \mathrm{~s}^{-1} \mathrm{~Pa}^{-1}$ and then slightly declines to $5.6 \times 10^{-7} \mathrm{~mol} \mathrm{~m}^{-2} \mathrm{~s}^{-1} \mathrm{~Pa}^{-1}$ after reaching a steady state shortly within $10 \mathrm{~h}$. The decreased permeance for nHEX is caused by an expansion of crystal lattice in the MFI zeolite [3], resulting in the permeation of some large molecules such as $23 \mathrm{DMB}$ and $22 \mathrm{DMB}$ through the membrane (Fig. 6). In spite of this, high separation factors of 16.6, 14.7, 18.3 and 23.6 for nHEX over 2MP, $3 \mathrm{MP}, 23 \mathrm{DMB}$, and $22 \mathrm{DMB}$ at the plateau confirm that the membrane is free of pinholes and cracks. Both permeance and separation factor remain constant after an execution of $72 \mathrm{~h}$, highlighting the high stability of this membrane.

\section{CONCLUSIONS}

In summary, we reported an advanced synthesis method

Table 1 Permeances of nHEX, 2MP, 3MP, 23DMB and 22DMB vapors and separation factors of nHEX over its isomers for the 500-nm-thick MFI zeolite membrane measured at $100^{\circ} \mathrm{C}$ with 1.2 bar feed gas

\begin{tabular}{|c|c|c|c|c|c|}
\hline & Molecular diameter $(\AA ̊)$ & Permeance & $\left.\mathrm{s}^{-1} \mathrm{~m}^{-2} \mathrm{~Pa}^{-1}\right)$ & \multicolumn{2}{|c|}{ Separation factor } \\
\hline nHEX & 4.3 & $24.5( \pm 1.1)^{\mathrm{a}}$ & $10.1( \pm 0.9)^{\mathrm{b}}$ & & \\
\hline $2 \mathrm{MP}$ & 5.4 & $1.79( \pm 0.2)^{\mathrm{a}}$ & $0.64( \pm 0.08)^{\mathrm{b}}$ & $13.7( \pm 0.8)^{\mathrm{a}}$ & $15.7( \pm 3.3)^{b}$ \\
\hline $3 \mathrm{MP}$ & 5.4 & $1.84( \pm 0.3)^{\mathrm{a}}$ & $0.69( \pm 0.08)^{\mathrm{b}}$ & $13.3( \pm 2.7)^{\mathrm{a}}$ & $14.7( \pm 2.9)^{\mathrm{b}}$ \\
\hline $23 \mathrm{DMB}$ & 5.8 & $1.14( \pm 0.2)^{\mathrm{a}}$ & $0.45( \pm 0.06)^{\mathrm{b}}$ & $21.5( \pm 4.4)^{\mathrm{a}}$ & $22.6( \pm 4.9)^{\mathrm{b}}$ \\
\hline $22 \mathrm{DMB}$ & 6.3 & $1.09( \pm 0.1)^{\mathrm{a}}$ & $0.33( \pm 0.04)^{\mathrm{b}}$ & $22.6( \pm 3.0)^{\mathrm{a}}$ & $30.5( \pm 6.2)^{b}$ \\
\hline
\end{tabular}

a), b) For single and binary (50:50 in volume) components, respectively. 


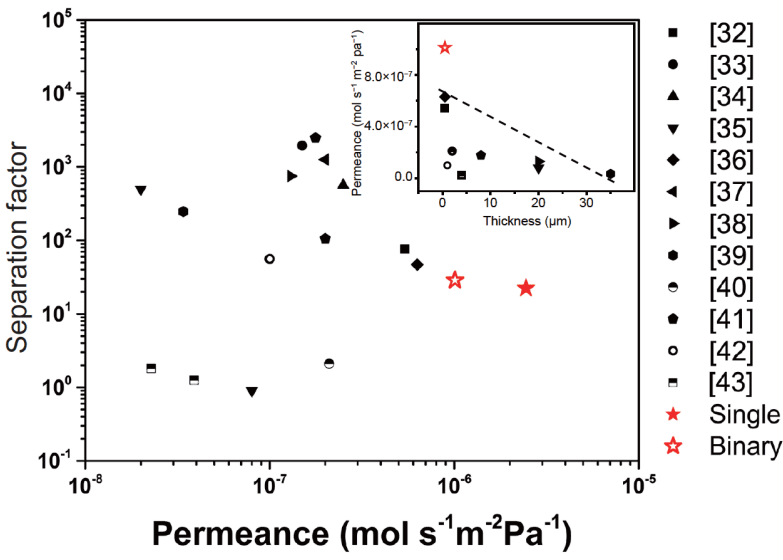

Figure 5 A summary of separation performances in terms of nHEX/ 22DMB separation factor and nHEX permeance for relevant polycrystalline zeolite membranes (the black symbols are the results from previous reports and the red stars refer to the results for single and binary gases in this study). The inset is the plot of the nHEX permeance versus the membrane thickness.

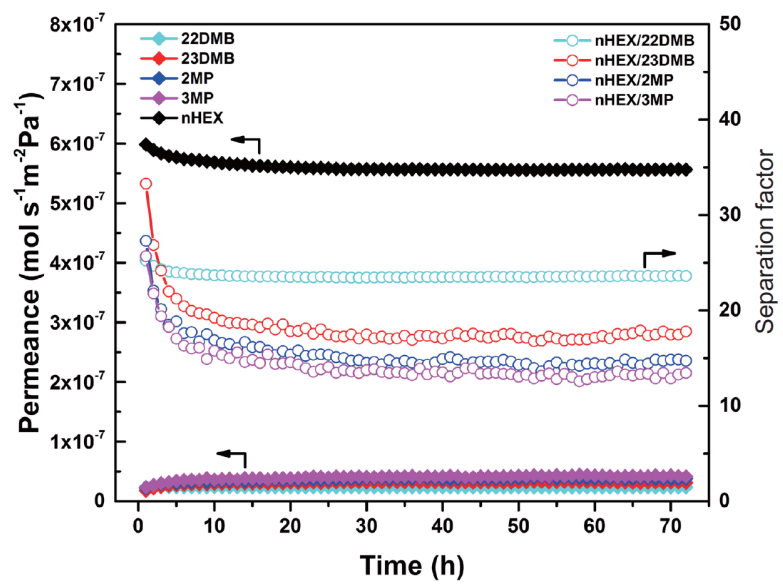

Figure 6 Permeances of nHEX, 2MP, 3MP, 23DMB and 22DMB, and separation factors of nHEX over its isomers for a mixture (13:20:18:19:30 in volume for nHEX:2MP:3MP:23DMB:22DMB) as a function of test time for the 500-nm-thick MFI zeolite membrane measured at $100^{\circ} \mathrm{C}$ with 1.2 bar feed gas.

called surface gel conversion for sustainable fabrication of highly inter-grown pure-silica MFI zeolite membranes as thin as $500 \mathrm{~nm}$ on porous alumina supports. The chemical composition of the synthesized gel is determinative in decreasing its wettability on the support, which is crucial in the success of reducing the membrane thickness down to submicron level. The seeds and water vapor facilitate gel crystallization and crystal intergrowth. The thinnest MFI zeolite membrane exhibits a record high nHEX permeance exceeding $2.0 \times 10^{-6} \mathrm{~mol} \mathrm{~m}^{-2} \mathrm{~s}^{-1} \mathrm{~Pa}^{-1}$ and an impressive selectivity greater than 13.3 for nHEX over other isomers in the hexane separation. In addition to the efficiency in making high-quality membranes, this method has several advantages of facile operation, full materials utilization and no waste disposal, shedding light on possible transition of zeolite membrane chemistry to industrial separation through scalable, economic and reproducible production of high-performance zeolite membranes.

Received 5 April 2020; accepted 19 May 2020;

published online 10 August 2020

1 Zou X, Zhu G. Microporous Materials for Separation Membranes. Weinheim: Wiley-VCH, 2019

2 Baerlocher C, McCusker LB, Olson DH. Atlas of Zeolite Framework Types. Amsterdam: Elsevier, 2007

3 O'Brien-Abraham J, Lin JYS. Zeolite membrane separations. In: Dunne SR, Ed. Zeolites in Industrial Separation and Catalysis. Weinheim: Wiley-VCH, 2010

4 Liu Y, Li Y, Cai R, et al. Suppression of twins in $b$-oriented MFI molecular sieve films under microwave irradiation. Chem Commun, 2012, 48: 6782-6784

5 Liu Y, Qiang W, Ji T, et al. Uniform hierarchical MFI nanosheets prepared via anisotropic etching for solution-based sub-100-nmthick oriented MFI layer fabrication. Sci Adv, 2020, 6: eaay5993

6 Liu Y, Li Y, Yang W. Fabrication of highly $b$-oriented MFI film with molecular sieving properties by controlled in-plane secondary growth. J Am Chem Soc, 2010, 132: 1768-1769

7 Chen HL, Li YS, Yang JS, et al. Preparation of high selectivity silicalite-1 membranes by two-step in situ hydrothermal synthesis. Chin Sci Bull, 2011, 56: 3578-3582

8 Pham TCT, Nguyen TH, Yoon KB. Gel-free secondary growth of uniformly oriented silica MFI zeolite films and application for xylene separation. Angew Chem Int Ed, 2013, 52: 8693-8698

9 Lu X, Yang Y, Zhang J, et al. Solvent-free secondary growth of highly $b$-oriented MFI zeolite films from anhydrous synthetic powder. J Am Chem Soc, 2019, 141: 2916-2919

10 Tsapatsis M. Toward high-throughput zeolite membranes. Science, 2011, 334: 767-768

11 Zhou M, Korelskiy D, Ye P, et al. A uniformly oriented MFI membrane for improved $\mathrm{CO}_{2}$ separation. Angew Chem Int Ed, 2014, 53: 3492-3495

12 Wang X, Karakiliç P, Liu X, et al. One-pot synthesis of high-flux $b$ oriented MFI zeolite membranes for Xe recovery. ACS Appl Mater Interfaces, 2018, 10: 33574-33580

13 Yu L, Holmgren A, Hedlund J. A novel method for fabrication of high-flux zeolite membranes on supports with arbitrary geometry. J Mater Chem A, 2019, 7: 10325-10330

14 Sjöberg E, Sandström L, Öhrman OGW, et al. Separation of $\mathrm{CO}_{2}$ from black liquor derived syngas using an MFI membrane. J Membrane Sci, 2013, 443: 131-137

15 Cao Z, Zeng S, Xu Z, et al. Ultrathin ZSM-5 zeolite nanosheet laminated membrane for high-flux desalination of concentrated brines. Sci Adv, 2018, 4: eaau8634

16 Jeon MY, Kim D, Kumar P, et al. Ultra-selective high-flux membranes from directly synthesized zeolite nanosheets. Nature, 2017, 543: 690-694

17 Agrawal KV, Topuz B, Pham TCT, et al. Oriented MFI membranes 
by gel-less secondary growth of sub-100 nm MFI-nanosheet seed layers. Adv Mater, 2015, 27: 3243-3249

18 Yeo ZY, Chew TL, Zhu PW, et al. Synthesis and performance of microporous inorganic membranes for $\mathrm{CO}_{2}$ separation: A review. J Porous Mater, 2013, 20: 1457-1475

19 Kiyozumi Y, Nagase T, Hasegawa Y, et al. A process for synthesizing bilayer zeolite membranes. Mater Lett, 2008, 62: 436-439

20 Chen $\mathrm{P}$, Chen X, Chen X, et al. Preparation and catalytic activity of titanium silicalite-1 zeolite membrane with TPABr as template. J Membrane Sci, 2009, 330: 369-378

21 Pan S, Meng X, Wang Z, et al. An efficient synthesis of NaA zeolite membranes from direct crystallization of gel-dipped macroporous alumina tubes with seeds. J Mater Chem A, 2018, 6: 10484-10489

22 Kwon YH, Kiang C, Benjamin E, et al. Krypton-xenon separation properties of SAPO-34 zeolite materials and membranes. AIChE J, 2017, 63: 761-769

23 Li M, Zhang J, Liu X, et al. Synthesis of high performance SAPO34 zeolite membrane by a novel two-step hydrothermal synthesis + dry gel conversion method. Microporous Mesoporous Mater, 2016, 225: 261-271

24 Chai L, Yang J, Wang J, et al. Preparation of silicalite-2 zeolite membrane on macroporous support by seeded steam-assisted crystallization method. Chem Lett, 2015, 44: 1592-1594

25 Guo H, Zhu G, Li H, et al. Hierarchical growth of large-scale ordered zeolite silicalite-1 membranes with high permeability and selectivity for recycling $\mathrm{CO}_{2}$. Angew Chem Int Ed, 2006, 45: 70537056

26 Morrow BA, McFarlan AJ. Surface vibrational modes of silanol groups on silica. J Phys Chem, 1992, 96: 1395-1400

27 Gallas JP, Goupil JM, Vimont A, et al. Quantification of water and silanol species on various silicas by coupling IR spectroscopy and in-situ thermogravimetry. Langmuir, 2009, 25: 5825-5834

28 Biswas RK, Khan P, Mukherjee S, et al. Study of short range structure of amorphous silica from PDF using Ag radiation in laboratory XRD system, RAMAN and NEXAFS. J Non-Cryst Solids, 2018, 488: 1-9

29 Wang T, Ni MJ, Luo ZY, et al. Viscosity and aggregation structure of nanocolloidal dispersions. Chin Sci Bull, 2012, 57: 3644-3651

30 Yuan Y, Lee TR. Contact angle and wetting properties. In: Bracco G, Holst B, Eds. Surface Science Techniques. Heidelberg: Springer, 2013

31 Yu G, Zou X, Sun L, et al. Constructing connected paths between UiO-66 and PIM-1 to improve membrane $\mathrm{CO}_{2}$ separation with crystal-like gas selectivity. Adv Mater, 2019, 31: 1806853

32 Hedlund J, Sterte J, Anthonis M, et al. High-flux MFI membranes. Microporous Mesoporous Mater, 2002, 52: 179-189

33 Gade SK, Tuan VA, Gump CJ, et al. Highly selective separation of $n$-hexane from branched, cyclic and aromatic hydrocarbons using B-ZSM-5 membranes. Chem Commun, 2001, 37: 601-602

34 Arruebo M, Falconer JL, Noble RD. Separation of binary $\mathrm{C}_{5}$ and $\mathrm{C}_{6}$ hydrocarbon mixtures through MFI zeolite membranes. J Membrane Sci, 2006, 269: 171-176

35 Coronas J, Noble RD, Falconer JL. Separations of $\mathrm{C}_{4}$ and $\mathrm{C}_{6}$ isomers in ZSM-5 tubular membranes. Ind Eng Chem Res, 1998, 37: $166-176$

36 Jareman F, Hedlund J, Sterte J. Effects of aluminum content on the separation properties of MFI membranes. Sep Purif Technol, 2003, 32: $159-163$

37 Flanders C, Tuan VA, Noble RD, et al. Separation of $\mathrm{C}_{6}$ isomers by vapor permeation and pervaporation through ZSM-5 membranes.
J Membrane Sci, 2000, 176: 43-53

38 Gump CJ, Noble RD, Falconer JL. Separation of hexane isomers through nonzeolite pores in ZSM-5 zeolite membranes. Ind Eng Chem Res, 1999, 38: 2775-2781

39 Kalipcilar H, Gade SK, Noble RD, et al. Synthesis and separation properties of B-ZSM-5 zeolite membranes on monolith supports. J Membrane Sci, 2002, 210: 113-127

40 Funke HH, Argo AM, Falconer JL, et al. Separations of cyclic, branched, and linear hydrocarbon mixtures through silicalite membranes. Ind Eng Chem Res, 1997, 36: 137-143

41 Choi J, Ghosh S, King L, et al. MFI zeolite membranes from $a$ - and randomly oriented monolayers. Adsorption, 2006, 12: 339-360

42 Deng Z, Pera-Titus M, Guo Y, et al. Molecular sieving separation of hexane isomers within nanocomposite (B)-MFI-alumina hollow fiber membranes: A modeling study. Ind Eng Chem Res, 2010, 49: $11697-11707$

43 Bárcia PS, Ferreira A, Gascon J, et al. Zeolite Beta membranes for the separation of hexane isomers. Microporous Mesoporous Mater, 2010, 128: 194-202

44 Baudot A, Bournay L. Integration of MFI zeolite membranes in the light gasoline isomerisation process. Oil Gas Sci Technol, 2009, 64: 759-771

Acknowledgements This work was supported by the National Natural Science Foundation of China (21531003, 21501024 and 21971035), Jilin Scientific and Technological Development Program (20170101198JC and 20190103017JH), Jilin Education Office (JJKH20180015KJ), "111" Program (B18012), and open projects from the State Key Laboratory of Inorganic Synthesis \& Preparative Chemistry and State Key Laboratory of Heavy Oil Processing (2018-8, SKLOP201902003).

Author contributions Zou X conceived the idea, designed and supervised the experiment; Rong $\mathrm{H}$ carried out the experiment, analyzed the data and drafted the paper; Wang G and Yan J performed the SEM and Raman measurements; Zhu G helped the data interpretation and commented on the paper. All authors contributed to the paper writing.

Conflict of interest The authors declare that they have no conflict of interest.

Supplementary information Supporting data are available in the online version of the paper.

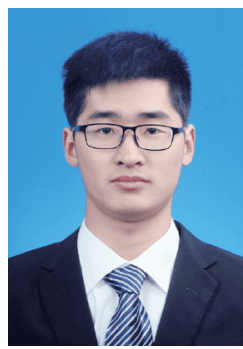

Huazhen Rong received his BSc degree in 2016 from Shandong Agricultural University, and joined Prof. Guangshan Zhu's research group in the same year. $\mathrm{He}$ is currently pursuing a $\mathrm{PhD}$ degree under the supervision of Prof. Xiaoqin Zou and Prof. Guangshan Zhu at Northeast Normal University. His research is centered on the synthesis and characterization of microporous membranes for separations. 


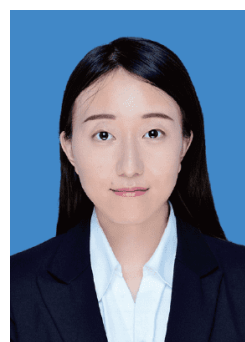

Gaimei Wang obtained her BSc degree in 2017 from Taiyuan Normal University. She is currently a master student under the supervision of Prof. Xiaoqin Zou at Northeast Normal University. Her current research interest focuses on the preparation and separation performance of microporous membranes.

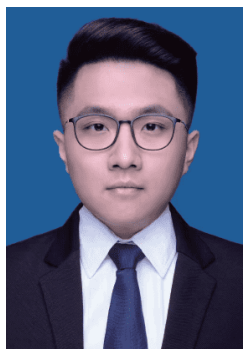

Jiaxu Yan received his BSc degree from Changchun University of Science and Technology in 2018. He is studying at Northeast Normal University for his MSc degree. His research interest focuses on the preparation and optical property modulation of $2 \mathrm{D}$ materials.

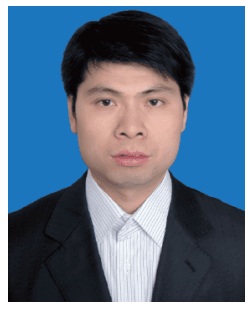

Xiaoqin Zou received his BSc and MSc degrees from Jilin University (2006 and 2009, respectively), and $\mathrm{PhD}$ degree (2012) from the University of Caen, France. He worked as a post-doc researcher during 2013-2015 in the University of California at Berkeley (USA). In 2015, he was appointed as a full professor in Northeast Normal University. His current scientific interests include the syntheses, characterizations and advanced applications of novel microporous materials.

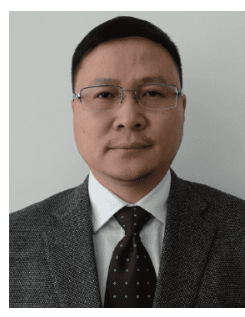

Guangshan Zhu studied chemistry in 1993 and earned his PhD degree at Jilin University in 1998. He worked as a post-doc fellow in 1999 at Tohoku University in Japan. He was a full professor since 2001 in Jilin University and moved to Northeast Normal University in 2015. The current research in his group focuses on the design and synthesis of porous materials, in particular, porous aromatic frameworks with applications in adsorption, separation and other areas.

表面胶转化法合成亚微米厚度MFI型分子篮薄膜 应用于快速择形分离己烷异构体

荣华臻 ${ }^{1}$, 王改梅 ${ }^{1}$, 间佳旭 ${ }^{2}$, 邹小勤 ${ }^{*}$, 朱广山 ${ }^{1}$

摘要 在膜分离中, 薄膜超薄化对促进气体传输至关重要, 而将膜 厚降至亚微米级则极具挑战性. 本文中, 我们提出表面胶转化法来 合成亚微米厚度的纯硅MFI分子篮膜. 通过精细调控前驱体化学组 成制备了低浸润性的黏性胶, 有效防止凝胶从氧化铝载体表面渗 透至空隙中. 在晶种的诱导下, 辅以少量水蒸气, 表面凝胶经历了 直接和完全晶化过程, 形成了厚度为 $500 \mathrm{~nm}$ 并且连续致密的MFI 分子篮膜. 合成的分子篮膜展示出对正已烷同分异构体的快速选 择性分离性能：超高的正已烷渗透量 $\left(24.5 \times 10^{-7} \mathrm{~mol} \mathrm{~m}^{-2} \mathrm{~s}^{-1} \mathrm{~Pa}^{-1}\right)$; 以及可观的分离系数(13.3-22.6, 正已烷对其同分异构体). 此外, 该 方法还是一种绿色合成方法, 为可持续性制备高质量分子篮膜奠 定了科学基础. 